\title{
Direct Evidence of Internalization of Tau by Microglia In Vitro and In Vivo
}

\author{
Marta Bolós ${ }^{\mathrm{a}, \mathrm{b}, 1}$, María Llorens-Martín ${ }^{\mathrm{a}, \mathrm{b}, 1}$, Jerónimo Jurado-Arjona ${ }^{\mathrm{a}, \mathrm{b}}$, Félix Hernández ${ }^{\mathrm{a}, \mathrm{b}}$, \\ Alberto Rábano ${ }^{c}$ and Jesús Avila ${ }^{\mathrm{a}, \mathrm{b}, *}$ \\ ${ }^{a}$ Centro de Investigación Biomédica en Red de Enfermedades Neurodegenerativas (CIBERNED), Madrid, Spain \\ bentro de Biología Molecular "Severo Ochoa" CSIC-UAM, Madrid, Spain \\ ${ }^{\mathrm{c}}$ Neuropathology Department, CIEN Foundation, Madrid, Spain
}

Accepted 8 October 2015

\begin{abstract}
The microtubule-associated protein (MAP) tau plays a critical role in the pathogenesis of tauopathies. Excess tau can be released into the extracellular medium in a physiological or pathological manner to be internalized by surrounding neuronsa process that contributes to the spread of this protein throughout the brain. Such spreading may correlate with the progression of the abovementioned diseases. In addition to neurons, tau can be internalized into other cells. Here we demonstrate that microglia take up tau in vitro and in vivo. In this regard, microglia from primary cultures internalized soluble (human recombinant tau42) and insoluble (homogenates derived from human AD brain) tau in vitro. Furthermore, using stereotaxic injection of tau in mice in vivo, we show that murine microglia internalize human tau. In addition, we demonstrate, for the first time, that microglia colocalize with various forms of tau in postmortem brain tissue of patients with Alzheimer's disease and non-demented control subjects. Our data reveal a potential role of microglia in the internalization of tau that might be relevant for the design of strategies to enhance the clearance of extracellular tau in neurodegenerative diseases characterized by the accumulation of this protein.
\end{abstract}

Keywords: Alzheimer's disease, clearance, microglia, tau protein, tauopathies

\section{INTRODUCTION}

Tau is a microtubule-associated protein that is toxic when present (mainly in its phosphorylated form) in high amounts inside neurons. In tauopathies, like Alzheimer's disease (AD), intracellular aggregates of phosphorylated tau are released to the extracellular space and propagate following a stereotypical pattern, from the transentorhinal and entorhinal cortex to the hippocampal formation and eventually to the neocortex. The spread of tau may contribute to cognitive decline in age-associated tauopathy $[1,2]$. In 2006, the notion of extracellular toxic tau protein was proposed

\footnotetext{
${ }^{1}$ These authors contributed equally to this work.

* Correspondence to: Jesús Avila de Grado, Centro de Biología Molecular 'Severo Ochoa', Universidad Autónoma de Madrid, C/Nicolás Cabrera 1, Madrid 28049, Spain. Tel.: +34 911964564; Fax: +34 911964420; E-mails: jesus.avila@csic.es; javila@cbm. csic.es.
}

[3]. Extracellular tau has also been detected in the brain interstitial fluid of both wild-type and P301S tau-overexpressing mice in microdialysis studies [4]. In addition, the levels of cerebrospinal fluid tau are elevated in $\mathrm{AD}$, as is also the case in patients with severe traumatic brain injury [5,6]. Tauopathies are characterized by massive accumulation of extracellular tau, mainly in an aggregated form. Studies performed in animals and in cell culture [7-11] suggest that tau aggregation propagates from neuron to neuron $[8,12]$.

Furthermore, it has been suggested that tau is secreted physiologically. Indeed, its physiological release was postulated as a way to prevent the toxicity caused by elevated intracellular tau [13, 14].

Microglia cells are the resident macrophages that constitute the main active immune cells in the brain. These cells play a continuous role of surveillance and phagocytosis in the central nervous system (CNS) and provide the initial response to CNS damage. Increasing 
evidence supports the notion that microglia-mediated neuroinflammatory processes directly contribute to the pathogenesis of AD. In this regard, neuroinflammation has been implicated in driving the hyperphosphorylation and aggregation of tau and neurodegeneration and recently in various models of tauopathies [15-23]. Nevertheless, it is unknown whether brain inflammation in $\mathrm{AD}$ patients is the cause of the disease or a secondary phenomenon.

Microglia colocalize with amyloid plaques in $\mathrm{AD}$ brains. It is been demonstrated that microglia are one of the major determinants of brain amyloid- $\beta(A \beta)$ clearance and amyloid burden $[24,25]$. In $A D$, microglia are able to bind to soluble $A \beta$ oligomers and $A \beta$ fibrils via cell surface receptors, including SCARA1, CD36, CD14, $\alpha 6 \beta 1$ integrin, CD47, and Toll-like receptors, and this process is thought to be part of the inflammatory reaction in $\mathrm{AD}[26,27]$. Generally, microglia are believed to clear $A \beta$ at early stages of $\mathrm{AD}$, but as the disease progresses, the ability of phagocytosis decreases due to reduced expression of their $A \beta$-binding receptors and $A \beta$-degrading enzymes. However, microglia maintain their ability to produce proinflammatory cytokines induced by soluble $A \beta$ or $A \beta$ deposits $[28,29]$. Although the relationship between microglia and $A \beta$ clearance is well established, little is known about the contribution of these cells to the uptake/clearance of extracellular tau. Of note, therapeutic interventions that prevent the uptake of aggregate seeds into neurons or promote microglial clearance may arrest disease. It has recently been reported that microglia enhance the clearance of aggregated tau when passive immunization with an anti-tau monoclonal antibody is used in vitro $[30,31]$.

Here we analyzed human tau in the following three systems: microglia cell cultures exposed to human tau; mice subjected to stereotaxic injection of tau; and brain tissue obtained from $\mathrm{AD}$ patients and control subjects. Using primary cultures of microglia and mice, respectively, we unambiguously demonstrate that microglia internalize extracellular soluble and insoluble tau in vitro and in vivo. Furthermore, we demonstrate that microglia colocalize with neurofibrillary tangles (NFTs) in postmortem human brain tissue.

\section{MATERIALS AND METHODS}

\section{Protein purification}

The recombinant human tau isoform containing 2 $\mathrm{N}$-terminal inserts and 4 microtubule binding repeats (tau42: [32]) was isolated as described previously [33]. Briefly, the proteins isolated were characterized by gel electrophoresis, followed by staining with Coomassie blue. Purified tau was labeled with sulfoindocyanine Cy5 dye (GE Healthcare, UK), as described previously [34] following the manufacturers recommendations. Briefly, $1 \mathrm{ml}$ of recombinant tau protein $(2 \mathrm{mg} / \mathrm{ml})$ was mixed with a sample of $\mathrm{Cy} 5$ dye at room temperature for $1 \mathrm{~h}$ for coupling to fluorophore. To remove the excess of free dye, the mixture was dialyzed overnight at $4^{\circ} \mathrm{C}$ against PBS and filtrated through a Sephadex G50 column. Cy5-coupled tau protein was characterized by Western blotting with an antibody that recognizes the dye (GE Healthcare, UK). The Cy5 fluorescent tracer was used because, due to its autofluorescence characteristics, the internalization of labeled-tau 42 into the microglia is easier to follow than if other tags were used.

\section{Primary microglia culture}

Cerebral cortices from 2-day-old Wistar rats were dissected, stripped of meninges, and digested with $0.1 \%$ trypsin at $37^{\circ} \mathrm{C}$ in $\mathrm{HBSS}$ (Gibco, USA) medium for $10 \mathrm{~min}$. Trypsinization was stopped by the addition of DMEM/Glutamax 1\%/Pyruvate 1\% (Gibco, USA) culture medium supplemented with $10 \%$ fetal bovine serum (Gibco, USA), $100 \mathrm{U} / \mathrm{mL}$ penicillin, and $0.1 \mathrm{mg} / \mathrm{mL}$ streptomycin (Gibco, USA). The cell suspension was pelleted, resuspended in culture medium, and finally suspended into a single-cell suspension by repeated pipetting, followed by passage through a 40$\mu \mathrm{m}$ pore mesh. Cells were seeded into a $75-\mathrm{ml}$ flask and cultured in medium at $37^{\circ} \mathrm{C}$ in humidified $5 \%$ CO2-95\% air. The culture medium was changed after 3 days. Floating microglia were recovered after 10-12 days by mild shaking and centrifugation and then were seeded onto 24 -well plates $\left(3 \times 10^{4}\right.$ cells/well $)$ with coverslips. Adherent cells were incubated for $48 \mathrm{~h}$ before being used for the experiments. Serum-free medium was used for the experiments. Cell specificity was determined using the microglial marker Iba-1, a polyclonal antibody (1:1000, Innogenetics, Belgium), and the astroglial marker GFAP (1:2000, Promega, USA). Cultured primary microglia were more than $98 \%$ positive for Iba- 1 .

\section{Tau internalization by microglia}

For the soluble tau experiments, primary microglia were treated with tau-Cy5 or vehicle (phosphate 
saline buffer (PBS)-Cy5) at $1 \mu \mathrm{M}$ for a range of times (5 min, $15 \mathrm{~min}, 1 \mathrm{~h}$, and $6 \mathrm{~h}$ ). In the case of insoluble tau, cells were treated with homogenized AD brain (tau-agg) or control PBS, following the same protocol. After treatment, cells were washed three times with PBS in order to remove the tau bound to the membrane. Cells were fixed with $4 \%$ paraformaldehyde in phosphate buffer for the immunofluorescence analysis. Each time-point was performed three times and the entire experiment was repeated independently three times $(n=3)$. A total of 50 cells per time were analyzed.

\section{Immunocytochemistry}

After treatment, cells were fixed in $4 \%(\mathrm{w} / \mathrm{v})$ paraformaldehyde in PBS. They were then stained with rabbit or goat anti-Iba1 (1:1000, Wako), rabbit anti-GFAP (1:2000, Promega), mouse antiCy5(1:2000, GE Healthcare), and rabbit anti-tau (1:1000, Novus, USA), all used diluted in $1 \%(\mathrm{v} / \mathrm{v})$ bovine serum plus $1 \%(\mathrm{v} / \mathrm{v})$ triton in PBS. They were then incubated with a donkey anti-goat IgG conjugated to Alexa Fluor 488, donkey anti-rabbit IgG conjugated to Alexa Fluor 488 or 555, donkey anti-mouse IgG conjugated to Alexa Fluor 647 (1:1000 diluted in 10\% $(\mathrm{v} / \mathrm{v})$ bovine serum plus $1 \%(\mathrm{v} / \mathrm{v})$ triton in PBS) and DAPI at 1:10,000 dilution. Iba-1, Cy5, and DAPIlabeled cells were counted under a $40 \times$ objective in five random fields throughout the coverslips. Confocal stacks of images were obtained (Z-axis interval: $1 \mu \mathrm{m})$, and z-projections were analyzed to determine the colocalization of Iba-1 and Cy5. Using ImageJ, we analyzed total internalized tau in thresholded images (threshold value $=50 ; 255$ ) as the percentage of Cy5 fluorescence intensity inside the cell normalized to that of total Iba1. For the statistical analysis, the total internalized Cy5-tau was related to the internalized Cy5 (control) at each time-point. A total of 50 cells per time were analyzed.

\section{Animals}

2-month-old C57B1/6 (Harlan Laboratories, Netherlands) mice were used for the in vivo experiments involving stereotaxic injection of tau. Mice were housed in a specific pathogen-free colony facility, in accordance with European Community Guidelines (directive 86/609/EEC), and handled following European and local animal care protocols. Animal experiments received the approval of the CBMSO's Ethics Committee. Four mice per experimental condition were used. Mice were left undisturbed for two weeks after arrival. Animals were 10 weeks old when the stereotaxic injections were performed.

\section{Stereotaxic surgery and sacrifice}

Mice were anesthetized with Isoflouran and placed in a stereotaxic frame. Coordinates $(\mathrm{mm})$ relative to the Bregma in the anteroposterior, mediolateral, and dorsoventral planes were as follows: dentate gyrus (DG) $[-2.0,1.4,2.2] .2 \mu 1 / \mathrm{DG}$ of tau-Cy5 or tauaggregate (tau-agg) solution was infused at $0.2 \mu \mathrm{l} / \mathrm{min}$ via a glass micropipette. After one week, mice were fully anesthetized by means of an intraperitoneal injection of pentobarbital and transcardially perfused with saline followed by $4 \%$ paraformaldehyde in phosphate buffer (PB). Brains were removed and post-fixed overnight.

\section{Human subjects}

The use of human hippocampus brain tissue samples was coordinated by local Brain Bank (Banco de Tejidos CIEN, Madrid), following national laws and international ethical and technical guidelines on the use of human samples for biomedical research purposes. In all cases, brain tissue donation, processing, and use for research were in compliance with published protocols (Best Practices for Repositories [35]), which include informed consent for brain tissue donation from living donors and the approval of the whole donation process by an ethical committee.

\section{Preparation of brain tissue extracts}

Brain extracts were isolated by homogenizing the tissue in ice-cold buffer $(1: 10 \mathrm{w} / \mathrm{v})$ consisting of $20 \mathrm{mM}$ HEPES pH7.4, $100 \mathrm{mM} \mathrm{NaCl}, 50 \mathrm{mM} \mathrm{NaF}$, 5 mM EDTA, $1 \%$ Triton X-100, and protease inhibitors (Roche, Switzerland), plus $100 \times$ ortovanadate and $1 / 1000$ okadaic acid (Sigma-Aldrich, USA). The homogenate was centrifuged at $100,000 \times g$ for $30 \mathrm{~min}$, and the supernatant was stored at $-20^{\circ} \mathrm{C}$ until use.

\section{Immunohistochemistry and confocal analysis}

Sagittal brain sections were obtained on a Leica VT1200S vibratome (50- $\mu \mathrm{m}$ thick sections). Immunohistochemistry was performed as described previously [36]. For all the immunohistochemical analyses, series 
of brain slices were made up randomly of one section from every ninth. One series, containing 8-10 sections, was used for the immunohistochemical detection of Iba1 and tau. In human tissue, the following primary antibodies were used: mouse anti-phospho-tau (PHF-1 epitope) (1:250, kindly provided by Davis); mouse anti-phospho-tau (AT100) (1:100, Innogenetics); mouse anti-phospho-tau (AT180) (1:100, Innogenetics); rabbit anti-phospho-tau (Tau396) (1:500, Life Technologies, USA); rabbit anti-phospho-tau (Tau404) (1:500, Life Technologies); mouse antidephosphorylated tau (Tau1) (1:200, Chemicon, Massachusetts); mouse anti-human tau (HT7) (1:500, Thermo Scientific, Waltham, MA); mouse antiphospho-au (AT8) (1:100, Innogenetis); and goat antiIba1(1:500, Wako), all in 1\% (v/v) bovine serum plus $1 \%(\mathrm{v} / \mathrm{v})$ triton in PB $1 \mathrm{~N}$. In mice, the following antibodies were used: mouse anti-human tau (HT7) (1:500, Thermo Scientific, MA); mouse anti-Cy5 (1:2000, GE Healthcare); and goat anti-Iba1 (1:500, Wako), all in $1 \%(\mathrm{v} / \mathrm{v})$ bovine serum plus $1 \%(\mathrm{v} / \mathrm{v})$ triton in PB $1 \mathrm{~N}$. All primary antibodies were incubated for $48 \mathrm{~h}$ at $4{ }^{\circ} \mathrm{C}$. The following secondary Alexa-conjugated antibodies were used at a final concentration of 1:1,000 diluted in $1 \%(\mathrm{v} / \mathrm{v})$ bovine serum plus $1 \%(\mathrm{v} / \mathrm{v})$ triton in $\mathrm{PB}$ $1 \mathrm{~N}$ : donkey anti-mouse IgG conjugated to Alexa Fluor 555; donkey anti-rabbit IgG conjugated to Alexa Fluor 555; and donkey anti-goat IgG conjugated to Alexa Fluor 647 (Life Technologies). Secondary antibodies were incubated overnight at $4{ }^{\circ} \mathrm{C}$. All sections were counterstained with DAPI (Calbiochem, 1:5000). For human slices, immunohistochemistry was performed as described, adding an autofluorescence eliminator reagent (Millipore, USA) after DAPI incubation in order to remove possible autofluorescence of the tissue. Images were taken under $25 \mathrm{X}, 40 \mathrm{X}$, and $63 \mathrm{X}$ objectives. Confocal stacks of images were obtained (Z-axis interval: $1 \mu \mathrm{m}$ ), and $\mathrm{z}$-projections were analyzed to determine the colocalization of the Iba- 1 and tau stained.

\section{Statistical analysis}

Statistical analysis was performed with GraphPad Prism software, Version 5.01. Data were tested by twoway ANOVA. Post-hoc comparisons were analyzed using Tukey's test. Differences were considered statistically significant when the probability, $\mathrm{p}$, of the null hypothesis was $\leq 0.05$. Data are presented as the means \pm S.E. All results were obtained in at least three independent experiments.

\section{RESULTS}

\section{Microglia take up soluble and insoluble human tau in vitro}

It has been suggested that sarkosyl-insoluble tau is internalized by microglia [31]. To confirm these results, we used a primary microglia culture model and insoluble tau obtained from homogenates of postmortem AD brain tissue (tau-agg). As microglia do not have endogenous tau, the tau antibody used (rabbit anti-human tau) recognized only the exogenous form of the protein taken up by microglia. Microglia were incubated with tau-agg or control PBS. Representative z-stack and orthogonal view images of treated cells are shown in Fig. 1A-C. Tau (red) was not present inside Iba1-stained microglia cells (green) when these were treated with control PBS (Fig. 1A). However, when tau-agg was added to the culture medium, tau was detected as red puncta inside microglia (Fig. 1B,C). These results are consistent with the findings of previous studies [31], confirming that microglia derived from primary cultures have the capacity to take up insoluble tau.

To study whether soluble tau was also internalized by microglia, tau42 labeled with Cy5 (tau-Cy5), deficient in the formation of tau aggregates, or control PBS-Cy5 was added to primary microglia cultures at $1 \mu \mathrm{M}$ of final concentration (Fig. 1D,E). Tau (stained using an antibody against $\mathrm{Cy} 5$ ) was detected inside the microglia treated with tau-Cy5 (Fig. 1E) but not in the control (Fig. 1D). This observation indicates the absence of an effect of Cy5 on tau uptake.

Next, we examined whether the internalization of tau by microglia was time-dependent. Microglia were incubated with tau-Cy5 or control PBS-Cy5 for 0, 5, 15, 60, and $360 \mathrm{~min}$. After this time, we calculated the presence of internalized tau as the difference between the percentage of total Iba1 fluorescence intensity and the percentage of Cy5 fluorescence intensity inside the cell compared to the control (Fig. 1K). Representative orthogonal images for the different time points showed that tau colocalized with Iba1 (Fig. 1F-J). We found that as early as 5 min after treatment, tau was detected inside microglia and the Cy5 fluorescence intensity progressively increased during the incubation time, up to the last time point of $360 \mathrm{~min}$. After $6 \mathrm{~h}$ of treatment with tau-Cy5, but not with control PBS-Cy5, we detected a high mortality of microglia. This observation could be attributed to a toxic effect of tau on these cells. We were therefore not able to quantified cells in the further times and consequently set $6 \mathrm{~h}$ as the 

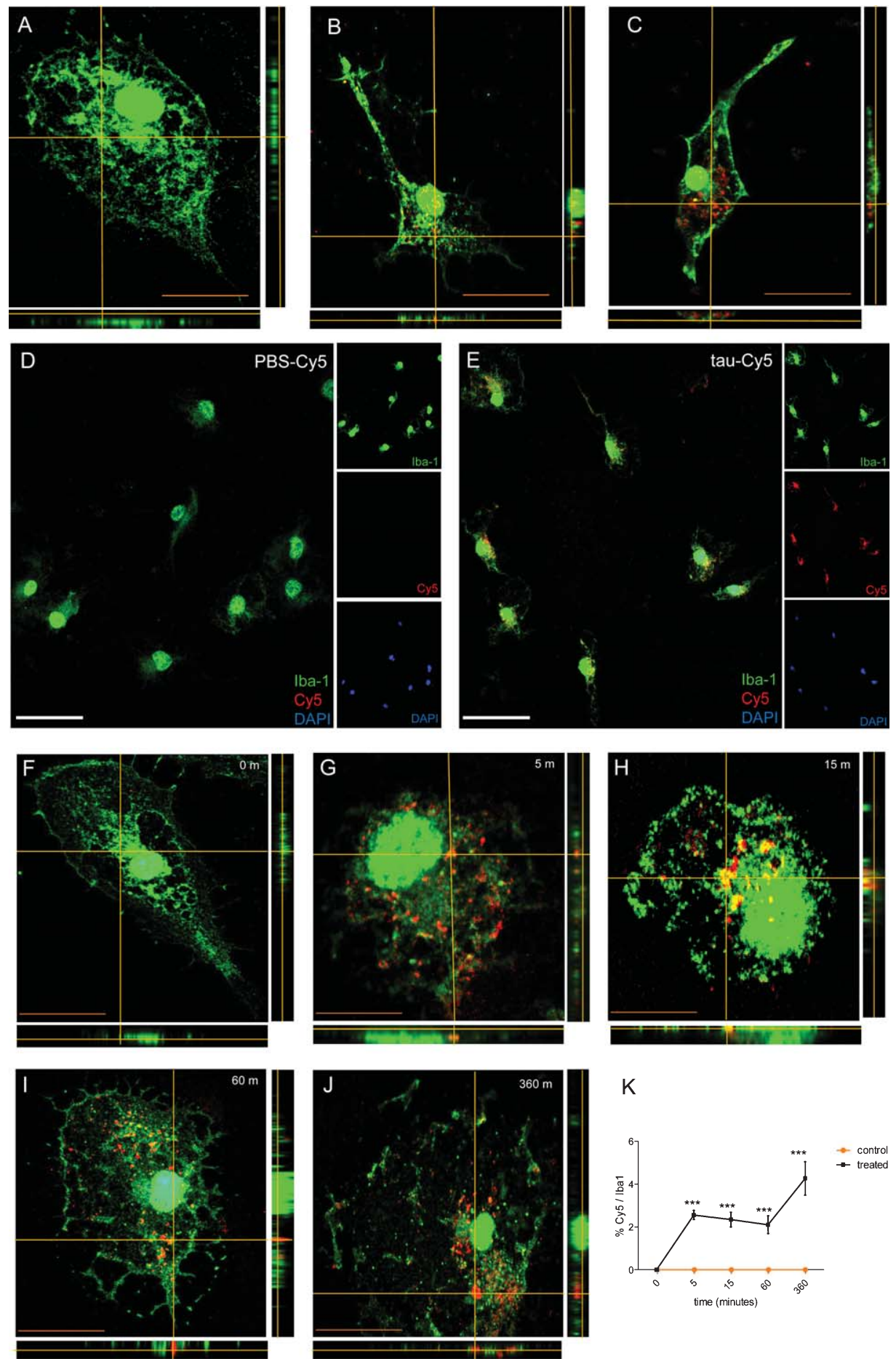

Fig. 1. Microglia internalized extracellular tau in vitro. Microglia derived from primary cultures were incubated with insoluble tau-agg (A-C) or soluble tau-Cy5 (D-K). Panels A-C show representative immunofluorescence images of microglia stained for Iba1 (green) and tau (red). The cultures were either untreated (A) or treated with $1 \mu \mathrm{M}$ tau-agg (B,C). Panels D and E show representative images of microglia culture control (D) or treated with $1 \mu \mathrm{M}$ tau-Cy5. Immunofluorescence images for Iba1 (green), Cy5 (red), and DAPI are shown separately. Panels F-J show representative immunofluorescence images of microglia (Iba1, green) treated with $1 \mu \mathrm{M}$ tau-Cy5 (Cy5, red) for different times (0-360 min). K) Quantification of results from immunofluorescence analysis showing \% Cy5/Iba1 fluorescence intensity present in cells treated with tau-Cy5 relative to the control Cy5. Bars show means \pm S.E. ${ }^{* * *} p \leq 0.001$. Orange scale bar: $10 \mu \mathrm{m}$, White scale bar: $50 \mu \mathrm{m}$. 

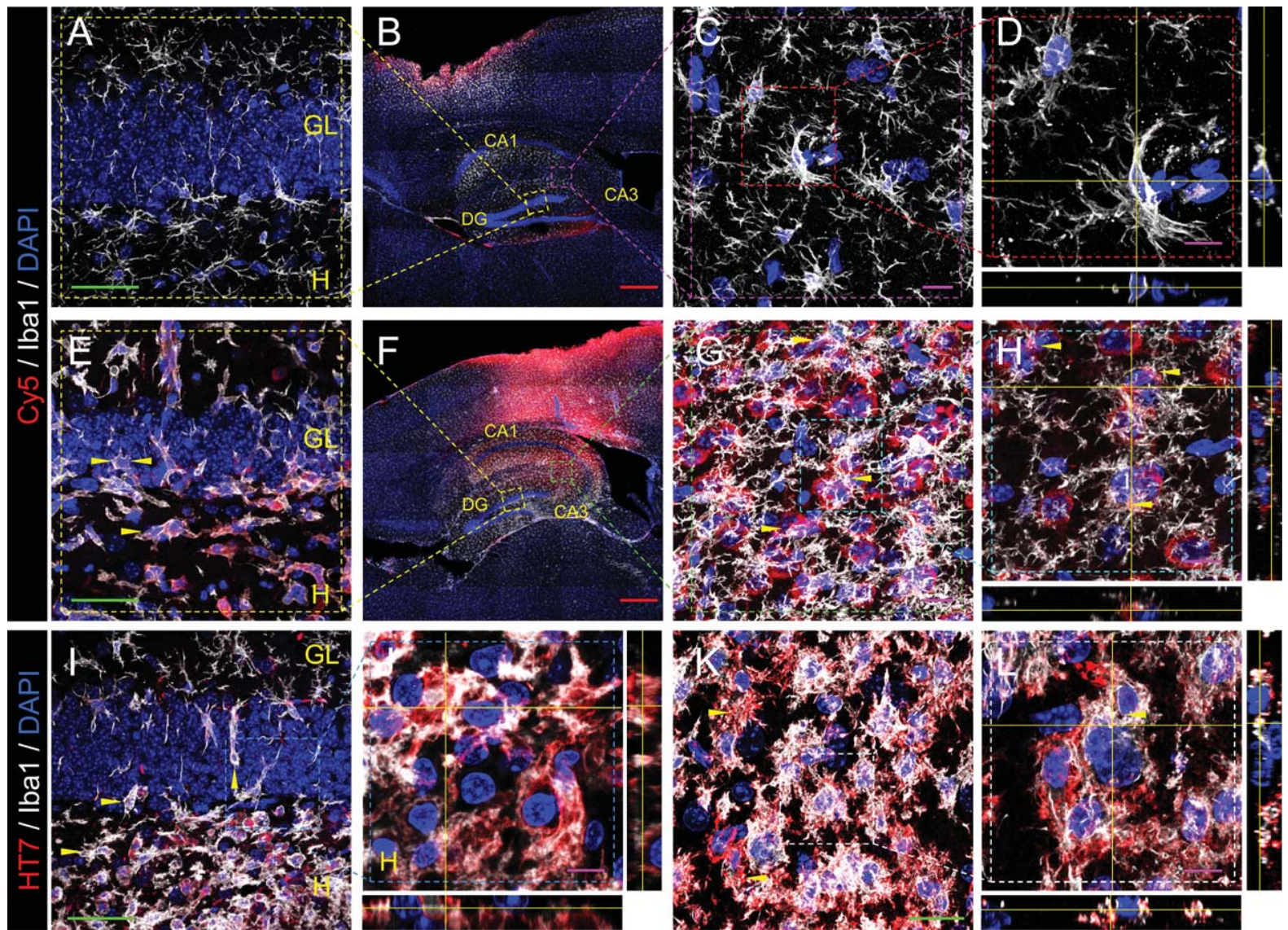

Fig. 2. Murine microglia internalized human tau in vivo. Representative images showing microglia labeled with Iba1 (white), human tau labeled with anti-Cy5 antibody, an anti-human tau antibody (HT7) (red), and DAPI (blue) in tissue derived from C57B1/6J mice stereotaxically injected with PBS-Cy5 (A-D) and $1 \mu \mathrm{M}$ of tau-Cy5 (E-H) or tau-agg (I-L). B and F) Tile Scan acquisition showing the whole anatomy of the hippocampus in control (B) or tau-Cy5-injected (F) mice. A,E,I,J representative images show DG hippocampal region injected with control PBS-Cy5 (A), tau-Cy5 (E), or tau-agg (I) and its magnification (J). C,D,G,H,K and L show high-power magnifications of the CA3 hippocampal region. One week after injection, it can be observed Iba1 (white) colocalization with both Cy5 (G) and HT7 (K), but not with PBS-Cy5 (C), in the hippocampus and the magnification images of PBS-Cy5 (D), tau-Cy5 (H) and tau-agg (L). DG, dentate gyrus. GL, granular layer. Yellow triangles indicate the presence of microglia and tau colocalization. Magenta scale bar: $10 \mu \mathrm{m}$. Green scale bar: $50 \mu \mathrm{m}$. Red scale bar: $200 \mu \mathrm{m}$.

Fig. 3. Microglia and tau colocalization in human AD brain tissue. Representative images showing microglia labeled with Iba1 (white), and tau labeled with different anti-human tau antibodies (red) and DAPI (blue) in tissue derived from the brains of a human AD patient (A-I) and a control subject $(\mathrm{J}-\mathrm{O})$. In order to analyze the colocalization of Iba1 and Tau staining, orthogonal views have been provided for each Z-projection at the right and bottom of each photomicrograph. It should be noted that the orthogonal views correspond to one single plane. It is possible to observe colocalization of the red and white channels indicated by the intersections between yellow. Various tau antibodies were used to detect tau in AD brain: anti-phospho-tau (PHF-1 epitope) in panel A and its magnification in B; anti-phospho-tau (AT100) in panel C; anti-phospho-tau (AT180) in panel F; anti- phospho-tau (Tau396) in panel D and its magnification in E; anti- phospho-tau (Tau404) in panel G; anti-de-phosphorylated tau (Tau1) in panel H; and anti-total human tau (HT7) in panel I. In control brains, representative images of the following anti-human tau antibodies are shown: anti- phospho-tau (AT180) in panel J; anti- phospho-tau (AT8) in panel K; anti-phospho-tau (Tau396) in panel L, and anti- phospho-tau (Tau404) in panels M-O. It can be observed how aberrant tau-labeled structures are much more abundant in the brain of the AD patient than in that of the control subject. It should be noted that independently of the phosphorylation state of tau, microglia show close contact with tau-labeled structures, thus suggesting that these cells play an active surveillance role aimed to control the progression of these aberrant structures. Green asterisks indicate the presence of tangles. Yellow triangles indicate microglia and tangle colocalization. Blue scale bar: $10 \mu \mathrm{m}$. Green scale bar: $50 \mu \mathrm{m}$. 

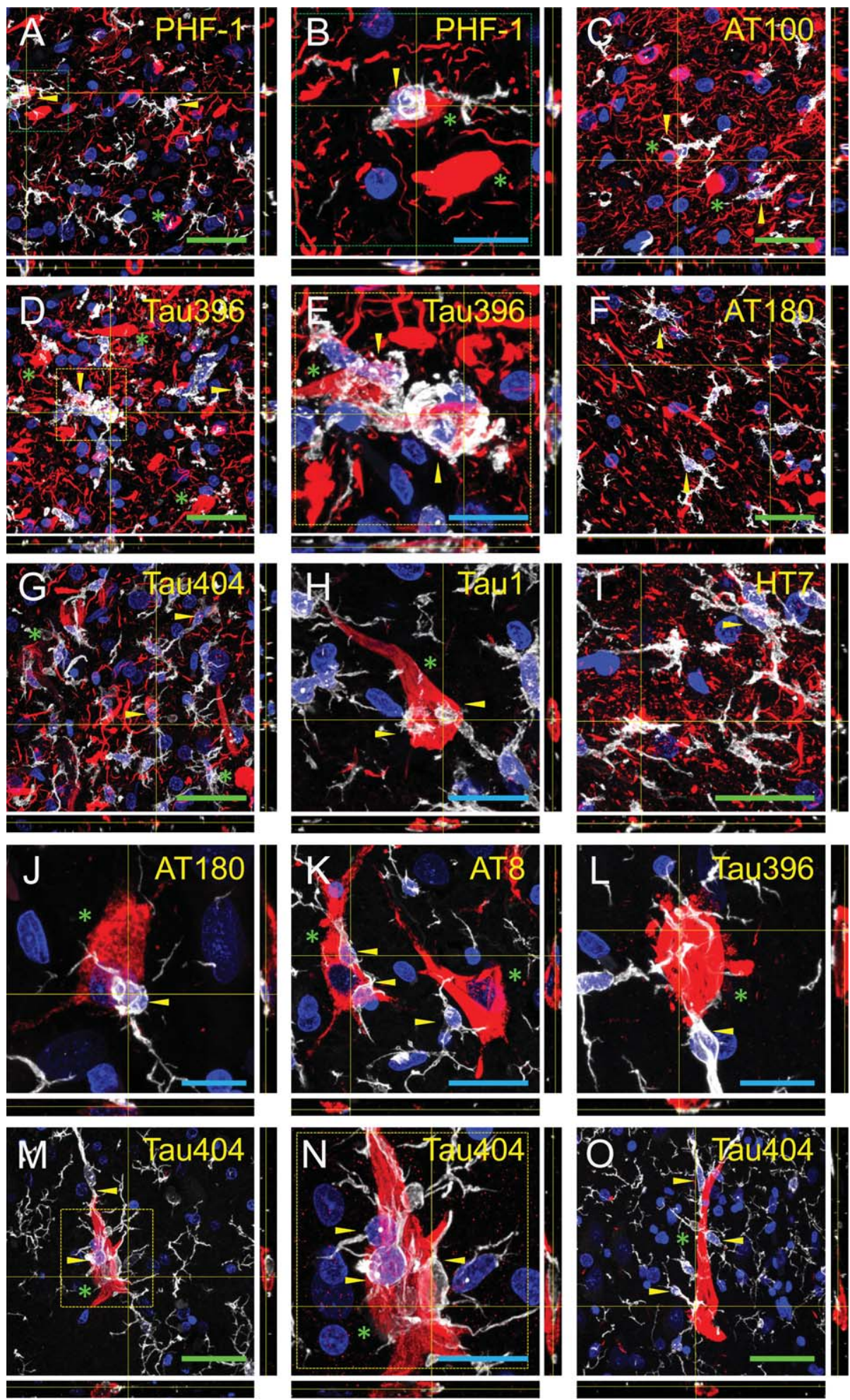
last experimental time point for our tau internalization study.

\section{Extracellular human tau was internalized by microglia in vivo}

As our results suggested that extracellular tau was internalized by microglia in primary cultures, we next examined whether murine microglia takes up human tau in vivo. With this aim, both control PBS-Cy5 (Fig. 2A-D) and soluble tau (tau-Cy5) (Fig. 2E-H) were injected into the brains of $\mathrm{C} 57 \mathrm{Bl} / 6$ mice. After one week, hippocampal brain slices were examined. A panoramic view showing the pattern of staining with an anti Cy5 antibody after the injections can be observed in control (Fig. 2B) and tau-Cy5 injected (Fig. 2F) mice. When soluble tau was injected (Fig. 2E-H), Iba1-labeled microglia (white) colocalized with Cy5labeled tau (red), in the dentate gyrus (Fig. 2E) and CA3 (Fig. 2G,H) hippocampal fields. Note in the high-power magnification of this image (Fig. 2E,G,H), marked with yellow triangles, that the colocalization between $\mathrm{Iba}^{+}$and $\mathrm{Cy}^{+}$occurred in all the $\mathrm{Iba}^{+}$ cells in the area. Orthogonal view of the magnification image showed how tau clearly colocalized with microglia (Fig. 2H). Thus, we concluded that microglia have the capacity to internalize soluble human tau in vivo.

In addition, we injected insoluble tau form (tau-agg) following the same protocol. The presence of tau was detected with the HT7 anti-human tau antibody (red). Intense staining of HT7 inside the $\mathrm{Iba}^{+}$microglia (white) was detected in the dentate gyrus (Fig. 2I,J) and CA3 (Fig. 2K,L) hippocampal subfields. These results showed, for the first time, that mouse microglia efficiently recognize and internalize free tau in vivo regardless of whether it is present in its soluble or insoluble form.

\section{Microglia colocalized with tau in human brain}

One of the hallmarks of AD are NFTs, which comprise aggregates of hyperphosphorylated tau. Although the presence of microgliosis in $\mathrm{AD}$ is well known, no study has demonstrated that microglia is in direct apposition of tau in human tissue. To address this question, we next examined whether microglia colocalize with tau in postmortem tissue derived from human AD and control brains and studied the threedimensional organization of these putative contacts. Tau was detected using several antibodies. Independently of the phosphorylation state of the protein, all these antibodies distinguished tau accurately in the two types of brain tissue. Nevertheless, the presence of tau was more accentuated in the AD brain (Fig. 3A-I versus Fig. 3J-O). In line with this, various antibodies against phosphorylated tau showed high levels of phospho-tau in tissue derived from human AD brain: anti-PHF1 (Fig. 3A,B), anti-Tau396 (Fig. 3D,E), anti-AT100 (Fig. 3C), anti-AT180 (Fig. 3F), and anti-Tau404 (Fig. 3G). Interestingly, low levels of phospho-tau were also detected in tissue derived from human control brain: anti-AT180 (Fig. 3J), anti-AT8 (Fig. 3K), anti-Tau396 (Fig. 3L), and anti-Tau404 (Fig. 3M-O). We found that tangles, marked with green asterisks, were labeled with all of the antibodies used. Accordingly, more tangles were present in the $\mathrm{AD}$ brain as compared to control subjects. Furthermore, these tangles and other tau ${ }^{+}$structures were found under close surveillance by Iba1-labeled microglia cells (Fig. 3A-O). We observed direct apposition of microglia to different types of $\mathrm{tau}^{+}$structures, including NFTs and dystrophic neurites. Microglia and aberrant tau ${ }^{+}$structures were found to be in close contact (yellow triangles in Fig. 3A,C,D,F,G). In tissue derived from control brain, scattered tangles were found in some hippocampal regions. In these areas, microglia (white) also colocalized with tangles (red). Green asterisks indicate the direct overlay between microglia and these tau ${ }^{+}$NFT (yellow triangles) (Fig. 3J-O). In addition, microglia colocalized not only with phosphorylated forms of tau, but also in those cases in which a total human-tau (HT7) antibody was used (Fig. 3I), and, importantly, they also showed a marked colocalization with de-phosphorylated forms of the protein, stained with an anti-Taul antibody (Fig. 3H). In summary, these results demonstrate that microglia colocalized with tau in human tissue of both $\mathrm{AD}$ patients and control subjects. On the basis of our observations, we propose that the surveillance role of microglia is driven by the presence of tau protein in aberrant structures such as NFTs and dystrophic neurites.

\section{DISCUSSION}

The present study shows that human extracellular tau is internalized by microglia in vitro and in vivo. We tested this internalization in three models, namely primary microglia culture exposed to tau as the in vitro model, C57B1/6 mice stereotaxically injected with tau, and human tissues from AD patients and control subjects for the in vivo studies. Soluble (monomeric or 
small aggregates) and insoluble (aggregates) human tau were used. Both forms of the protein were internalized by microglia derived from primary cultures. After intracerebral injection of tau in $\mathrm{C} 57 \mathrm{Bl} / 6$ mice, the two forms of the protein were also internalized by microglia. Furthermore, different forms of tau present in tissue derived from the postmortem brains of human AD patient and control subject colocalized with microglia. Taken together, these results demonstrate that microglia take up tau, regardless of the aggregation state of the protein, thereby supporting the notion that these cells play a key role in the clearance of tau.

A number of studies showing the release of extracellular tau by cell lines and neurons via multiple pathways, including cell death, strongly support the hypothesis that the secretion of tau is an important biological function of the protein, especially in disease $[37,38]$. In addition, increased intracellular levels of phosphorylated tau could be detrimental and would thus support the notion that secretion is a mechanism by which excess tau, mainly in the form of aggregates, is removed, thereby preventing the toxicity cause by its accumulation. This source of extracellular tau can be internalized by other cells, including neurons [39]. The spread of tau from cell to cell has been proposed to be one of the mechanisms underlying the progression of tau pathologies [12, 40, 41].

Therefore the inhibition of tau uptake by other cells or increasing microglial clearance of extracellular tau could be useful strategies to arrest these pathologies.

In addition, genome-wide association studies have recently found risk factors associated with a higher probability of developing sporadic AD. Two of these genes are TREM2 and CD33 [42-44], which are involved in the phagocytic and degradative functions of microglia. These genes play a key role in $\mathrm{A} \beta$ clearance by microglia [24, 45], a process that prevents the formation of amyloid plaques in AD. These findings suggest that microglial phagocytosis could be impaired in tauopathies, like $\mathrm{AD}$, thus contributing to the progression of the disease. In addition, recent evidence suggests that microglia participate in the internalization and degradation of pathological tau (insoluble) in vitro [30] and ex vivo [31], although the precise mechanisms remain unclear.

In primary cultures of microglia, we confirmed that extracellular insoluble human tau is internalized. These results are consistent with the findings of previous studies. Nevertheless, the most novel finding of the present work is that that primary cultures of microglia also take up soluble tau. Although soluble tau is consid- ered to be non-toxic, we observed high mortality of microglia that took up tau during a 6-h treatment. This observation could be attributable to a loss of the proteostasis of the cell. The role played by microglia in the uptake of tau was confirmed by in vivo studies. Therefore, the colocalization of $\mathrm{Iba}^{+}$microglia and stereotaxically-injected tau was analyzed in the hippocampus of C57BL/6 one week after the injection. Coincident with the in vitro results, we found that the microglia present in the hippocampus colocalizes with tau-Cy5 (detected with an anti-Cy5 antibody) as well as with tau-agg (detected with an anti-human tau antibody (HT7)). In addition and as expected, we found that the presence of tau (tangles and other aberrant structures) was enormously increased in human $\mathrm{AD}$ brains compared to the control subjects. Again, we observed microglia colocalizing with tau. Microglia were in close contact with aberrant structures in both AD patients and control subjects. These data strongly suggest that microglia play a crucial role in the surveillance of the aberrant process of NFT formation and in tau clearance under both physiological and pathological conditions.

In summary, using in vitro and in vivo models, here we demonstrate that microglia take up tau independently of the aggregation state of the protein. In addition, we show, for the first time, that microglia internalize extracellular tau in murine and human in vivo models. All together these results point to these cells as potential therapeutic targets in tauopathies.

\section{ACKNOWLEDGMENTS}

This study was funded by grants from the Spanish Ministry of Health, the Comunidad de Madrid, the Centro de Investigación Biomédica en Red sobre Enfermedades Neurodegenerativas (CIBERNED, ISCIII), and the Alzheimer's Association.

The authors thank R. Cuadros for help in producing Cy5-tau.

Authors' disclosures available online (http://j-alz. com/manuscript-disclosures/15-0704r1).

\section{REFERENCES}

[1] Braak H, Braak E (1991) Neuropathological stageing of Alzheimer-related changes. Acta Neuropathol 82, 239-259.

[2] Saito Y, Ruberu NN, Sawabe M, Arai T, Tanaka N, Kakuta Y, Yamanouchi H, Murayama S (2004) Staging of argyrophilic grains: An age-associated tauopathy. J Neuropathol Exp Neurol 63, 911-918. 
[3] Gomez-Ramos A, Diaz-Hernandez M, Cuadros R, Hernandez F, Avila J (2006) Extracellular tau is toxic to neuronal cells. FEBS Lett 580, 4842-4850.

[4] Yamada K, Cirrito JR, Stewart FR, Jiang H, Finn MB, Holmes BB, Binder LI, Mandelkow EM, Diamond MI, Lee VM, Holtzman DM (2011) in vivo microdialysis reveals age-dependent decrease of brain interstitial fluid tau levels in P301S human tau transgenic mice. J Neurosci 31, 13110-13117.

[5] Marklund N, Blennow K, Zetterberg H, Ronne-Engstrom E, Enblad P, Hillered L (2009) Monitoring of brain interstitial total tau and beta amyloid proteins by microdialysis in patients with traumatic brain injury. J Neurosurg 110, 1227-1237.

[6] Magnoni S, Esparza TJ, Conte V, Carbonara M, Carrabba G, Holtzman DM, Zipfel GJ, Stocchetti N, Brody DL (2012) Tau elevations in the brain extracellular space correlate with reduced amyloid-beta levels and predict adverse clinical outcomes after severe traumatic brain injury. Brain 135, 1268-1280.

[7] Clavaguera F, Bolmont T, Crowther RA, Abramowski D, Frank S, Probst A, Fraser G, Stalder AK, Beibel M, Staufenbiel M, Jucker M, Goedert M, Tolnay M (2009) Transmission and spreading of tauopathy in transgenic mouse brain. Nat Cell Biol 11, 909-913.

[8] Frost B, Jacks RL, Diamond MI (2009) Propagation of tau misfolding from the outside to the inside of a cell. $J$ Biol Chem 284, 12845-12852.

[9] Liu L, Drouet V, Wu JW, Witter MP, Small SA, Clelland C, Duff K (2012) Trans-synaptic spread of tau pathology in vivo. PLoS One 7, e31302.

[10] de Calignon A, Polydoro M, Suarez-Calvet M, William C, Adamowicz DH, Kopeikina KJ, Pitstick R, Sahara N, Ashe KH, Carlson GA, Spires-Jones TL, Hyman BT (2012) Propagation of tau pathology in a model of early Alzheimer's disease. Neuron 73, 685-697.

[11] Kfoury N, Holmes BB, Jiang H, Holtzman DM, Diamond MI (2012) Trans-cellular propagation of Tau aggregation by fibrillar species. J Biol Chem 287, 19440-19451.

[12] Holmes BB, Furman JL, Mahan TE, Yamasaki TR, Mirbaha H, Eades WC, Belaygorod L, Cairns NJ, Holtzman DM, Diamond MI (2014) Proteopathic tau seeding predicts tauopathy in vivo. Proc Natl Acad Sci U S A 111, E4376E4385.

[13] Simon D, Garcia-Garcia E, Royo F, Falcon-Perez JM, Avila J (2012) Proteostasis of tau. Tau overexpression results in its secretion via membrane vesicles. FEBS Lett 586, 47-54.

[14] Karch CM, Jeng AT, Goate AM (2012) Extracellular tau levels are influenced by variability in Tau that is associated with tauopathies. J Biol Chem 287, 42751-42762.

[15] Gebicke-Haerter PJ (2001) Microglia in neurodegeneration: Molecular aspects. Microsc Res Tech 54, 47-58

[16] Ishizawa K, Dickson DW (2001) Microglial activation parallels system degeneration in progressive supranuclear palsy and corticobasal degeneration. J Neuropathol Exp Neurol 60 , 647-657.

[17] Bellucci A, Bugiani O, Ghetti B, Spillantini MG (2011) Presence of reactive microglia and neuroinflammatory mediators in a case of frontotemporal dementia with P301S mutation. Neurodegener Dis 8, 221-229.

[18] Bellucci A, Westwood AJ, Ingram E, Casamenti F, Goedert M, Spillantini MG (2004) Induction of inflammatory mediators and microglial activation in mice transgenic for mutant human P301S tau protein. Am J Pathol 165, 1643-1652.
[19] Ikeda M, Shoji M, Kawarai T, Kawarabayashi T, Matsubara E, Murakami T, Sasaki A, Tomidokoro Y, Ikarashi Y, Kuribara H, Ishiguro K, Hasegawa M, Yen SH, Chishti MA, Harigaya Y, Abe K, Okamoto K, St George-Hyslop P, Westaway D (2005) Accumulation of filamentous tau in the cerebral cortex of human tau R406W transgenic mice. Am J Pathol 166, 521-531.

[20] Yoshiyama Y, Higuchi M, Zhang B, Huang SM, Iwata N, Saido TC, Maeda J, Suhara T, Trojanowski JQ, Lee VM (2007) Synapse loss and microglial activation precede tangles in a P301S tauopathy mouse model. Neuron 53, 337-351.

[21] Sasaki A, Kawarabayashi T, Murakami T, Matsubara E, Ikeda M, Hagiwara H, Westaway D, George-Hyslop PS, Shoji M, Nakazato Y (2008) Microglial activation in brain lesions with tau deposits: Comparison of human tauopathies and tau transgenic mice TgTauP301L. Brain Res 1214, 159-168.

[22] Zilka N, Stozicka Z, Kovac A, Pilipcinec E, Bugos O, Novak M (2009) Human misfolded truncated tau protein promotes activation of microglia and leukocyte infiltration in the transgenic rat model of tauopathy. J Neuroimmunol 209, 16-25.

[23] Maphis N, Xu G, Kokiko-Cochran ON, Jiang S, Cardona A, Ransohoff RM, Lamb BT, Bhaskar K (2015) Reactive microglia drive tau pathology and contribute to the spreading of pathological tau in the brain. Brain 138, 1738-1755.

[24] Jiang T, Yu JT, Hu N, Tan MS, Zhu XC, Tan L (2014) CD33 in Alzheimer's disease. Mol Neurobiol 49, 529-535.

[25] Herber DL, Mercer M, Roth LM, Symmonds K, Maloney J, Wilson N, Freeman MJ, Morgan D, Gordon MN (2007) Microglial activation is required for Abeta clearance after intracranial injection of lipopolysaccharide in APP transgenic mice. J Neuroimmune Pharmacol 2, 222-231.

[26] Heneka MT, Carson MJ, El Khoury J, Landreth GE, Brosseron F, Feinstein DL, Jacobs AH, Wyss-Coray T, Vitorica J, Ransohoff RM, Herrup K, Frautschy SA, Finsen B, Brown GC, Verkhratsky A, Yamanaka K, Koistinaho J, Latz E, Halle A, Petzold GC, Town T, Morgan D, Shinohara ML, Perry VH, Holmes C, Bazan NG, Brooks DJ, Hunot S, Joseph B, Deigendesch N, Garaschuk O, Boddeke E, Dinarello CA, Breitner JC, Cole GM, Golenbock DT, Kummer MP (2015) Neuroinflammation in Alzheimer's disease. Lancet Neurol 14, 388-405.

[27] Heppner FL, Ransohoff RM, Becher B (2015) Immune attack: The role of inflammation in Alzheimer disease. Nat Rev Neurosci 16, 358-372.

[28] Guillot-Sestier MV, Town T (2013) Innate immunity in Alzheimer's disease: A complex affair. CNS Neurol Disord Drug Targets 12, 593-607.

[29] Yu Y, Ye RD (2015) Microglial Abeta receptors in Alzheimer's disease. Cell Mol Neurobiol 35, 71-83.

[30] Funk KE, Mirbaha H, Jiang H, Holtzman DM, Diamond MI (2015) Distinct therapeutic mechanisms of tau antibodies: Promoting microglial clearance versus blocking neuronal uptake. J Biol Chem 290, 21652-32662.

[31] Luo W, Liu W, Hu X, Hanna M, Caravaca A, Paul SM (2015) Microglial internalization and degradation of pathological tau is enhanced by an anti-tau monoclonal antibody. Sci Rep $\mathbf{5}$, 11161.

[32] Goedert M, Jakes R (1990) Expression of separate isoforms of human tau protein: Correlation with the tau pattern in brain and effects on tubulin polymerization. EMBO J 9, 4225-4230.

[33] Perez M, Valpuesta JM, Medina M, Montejo de Garcini E, Avila J (1996) Polymerization of tau into filaments in the presence of heparin: The minimal sequence required for tautau interaction. $J$ Neurochem 67, 1183-1190. 
[34] Gomez-Ramos A, Diaz-Hernandez M, Rubio A, DiazHernandez JI, Miras-Portugal MT, Avila J (2009) Characteristics and consequences of muscarinic receptor activation by tau protein. Eur Neuropsychopharmacol 19, 708-717.

[35] (2012) 2012 best practices for repositories collection, storage, retrieval, and distribution of biological materials for research international society for biological and environmental repositories. Biopreserv Biobank 10, 79-161.

[36] Llorens-Martin M, Fuster-Matanzo A, Teixeira CM, JuradoArjona J, Ulloa F, Defelipe J, Rabano A, Hernandez F, Soriano E, Avila J (2013) GSK-3beta overexpression causes reversible alterations on postsynaptic densities and dendritic morphology of hippocampal granule neurons in vivo. Mol Psychiatry 18, 451-460.

[37] Kanmert D, Cantlon A, Muratore CR, Jin M, O’Malley TT, Lee G, Young-Pearse TL, Selkoe DJ, Walsh DM (2015) C-terminally truncated forms of tau, but not full-length tau or its C-terminal fragments, are released from neurons independently of cell death. J Neurosci 35, 10851-10865.

[38] Kim W, Lee S, Hall GF (2010) Secretion of human tau fragments resembling CSF-tau in Alzheimer's disease is modulated by the presence of the exon 2 insert. FEBS Lett 584, 3085-3088.

[39] Dujardin S, Lecolle K, Caillierez R, Begard S, Zommer N, Lachaud C, Carrier S, Dufour N, Auregan G, Winderickx J, Hantraye P, Deglon N, Colin M, Buee L (2014) Neuron-toneuron wild-type Tau protein transfer through a trans-synaptic mechanism: Relevance to sporadic tauopathies. Acta Neuropathol Commun 2, 14.

[40] Iba M, McBride JD, Guo JL, Zhang B, Trojanowski JQ, Lee VM (2015) Tau pathology spread in PS19 tau transgenic mice following locus coeruleus (LC) injections of synthetic tau fibrils is determined by the LC's afferent and efferent connections. Acta Neuropathol 130, 349-362.

[41] Holmes BB, DeVos SL, Kfoury N, Li M, Jacks R, Yanamandra K, Ouidja MO, Brodsky FM, Marasa J, Bagchi DP, Kotzbauer PT, Miller TM, Papy-Garcia D, Diamond MI (2013) Heparan sulfate proteoglycans mediate internalization and propagation of specific proteopathic seeds. Proc Natl Acad Sci U S A 110, E3138-E3147.

[42] Jonsson T, Stefansson H, Steinberg S, Jonsdottir I, Jonsson PV, Snaedal J, Bjornsson S, Huttenlocher J, Levey AI, Lah JJ, Rujescu D, Hampel H, Giegling I, Andreassen OA, Engedal K, Ulstein I, Djurovic S, Ibrahim-Verbaas C, Hofman A, Ikram MA, van Duijn CM, Thorsteinsdottir U, Kong A, Stefansson K (2013) Variant of TREM2 associated with the risk of Alzheimer's disease. N Engl J Med 368, 107-116.

[43] Guerreiro R, Wojtas A, Bras J, Carrasquillo M, Rogaeva E, Majounie E, Cruchaga C, Sassi C, Kauwe JS, Younkin S, Hazrati L, Collinge J, Pocock J, Lashley T, Williams J, Lambert JC, Amouyel P, Goate A, Rademakers R, Morgan K, Powell J, St George-Hyslop P, Singleton A, Hardy J, Alzheimer Genetic Analysis G (2013) TREM2 variants in Alzheimer's disease. N Engl J Med 368, 117-127.

[44] Bradshaw EM, Chibnik LB, Keenan BT, Ottoboni L, Raj T, Tang A, Rosenkrantz LL, Imboywa S, Lee M, Von Korff A, Alzheimer Disease Neuroimaging I, Morris MC, Evans DA, Johnson K, Sperling RA, Schneider JA, Bennett DA, De Jager PL (2013) CD33 Alzheimer's disease locus: Altered monocyte function and amyloid biology. Nat Neurosci 16, 848-850.

[45] Tahara K, Kim HD, Jin JJ, Maxwell JA, Li L, Fukuchi K (2006) Role of toll-like receptor signalling in Abeta uptake and clearance. Brain 129, 3006-3019. 